\title{
Note
}

\section{The impairment of emotion recognition in Huntington's disease extends to positive emotions}

\author{
Laura Robotham $^{a, b, c, 1}$, Disa A. Sauter ${ }^{d, 1}$, Anne-Catherine Bachoud-Lévi ${ }^{a, b, c, e, 2, *}$ \\ and Iris Trinkler $a, b, c, 2$ \\ ${ }^{a}$ INSERM U955, E1, Equipe de Neuropsychologie Interventionnelle, Institut Mondor de Recherche Biomédicale, Créteil, France \\ b Département d'Etudes Cognitives, Ecole Normale Supérieure, Paris, France \\ ${ }^{\mathrm{C}}$ Université Paris Est, Faculté de Médecine, Créteil, France \\ $\mathrm{d}$ The Max Planck Institute for Psycholinguistics, Nijmegen, The Netherlands \\ e AP-HP, Centre de Référence Maladie de Huntington, Service de Neurologie, CHU Henri Mondor, Créteil, France
}

\section{A R T I C L E I N F O}

Article history:

Received 1 October 2010

Reviewed 14 December 2010

Revised 26 December 2010

Accepted 14 February 2011

Action editor Paolo Nichelli

Published online 23 February 2011

\section{Keywords:}

Huntington's disease

Emotion

Vocalizations

Affect

\begin{abstract}
A B S T R A C T
Patients with Huntington's Disease (HD) are impaired in the recognition of emotional signals. However, the nature and extent of the impairment is controversial: it has variously been argued to disproportionately affect disgust (e.g., Sprengelmeyer et al., 1996), to be general for negative emotions (Snowden et al., 2008), or to be a consequence of item difficulty (Milders et al., 2003). Yet no study to date has included more than one positive stimulus category in emotion recognition tasks, and most studies have focused on the recognition of emotions from facial stimuli. In this study, we test the hypothesis that patients with HD may be impaired in their recognition of positive as well as negative emotional signals, by examining the recognition of a range of positive emotions from vocal cues. We present a study of 14 Huntington's patients and 15 controls performing a forcedchoice task with a previously validated set of negative and positive non-verbal emotional vocalizations (Sauter and Scott, 2007). Although HD patients performed above chance for each emotion, they were found to be impaired in both positive and negative emotions, including pleasure, fear and anger. These findings complement previous work by demonstrating that impairments in emotion recognition in HD extend to positive and negative emotions, which may imply a general deficit.
\end{abstract}

(c) 2011 Elsevier Srl. All rights reserved.

\section{Introduction}

Huntington's disease (HD) is a rare, inherited, neurodegenerative disease characterized by motor impairments. It is associated with numerous cognitive and attentional deficits, as well as difficulty in processing and interpreting emotions. But although it is well established that patients with HD are impaired in the recognition of emotional signals, there is little agreement about the nature and extent of this impairment. Earlier studies found an impairment that disproportionately

\footnotetext{
* Corresponding author. Service de neurologie, Hôpital Henri Mondor, 51 av du Mal de Lattre de Tassigny, 94010 Créteil cedex, France. E-mail address: bachoud@gmail.com (A.-C. Bachoud-Lévi).

${ }^{1}$ These two authors contributed equally to this work.

2 These two authors contributed equally to this work. 0010-9452/\$ - see front matter @ 2011 Elsevier Srl. All rights reserved. doi:10.1016/j.cortex.2011.02.014
} 
affected the recognition of disgust expressions in clinical (e.g., Sprengelmeyer et al., 1996) and a circumscribed deficit for disgust in pre-clinical individuals with HD (e.g., Gray et al., 1997). However, several studies have documented a more general impairment encompassing several negative emotions in clinical patients (Calder et al., 2010) or virtually all negative emotions across both pre-clinical and clinical individuals (e.g., De Gelder et al., 2008; Johnson et al., 2007; Snowden, et al., 2008). Some authors have argued that the selective impairment for disgust stimuli found in some studies may have been a consequence of item difficulty (Milders et al., 2003). Recently, Snowden et al. (2008) employed ten different tasks assessing recognition of emotion from facial and vocal cues in patients with manifest HD. They found that patients were impaired in the recognition of several negative emotions across their extensive battery of tasks and concluded that HD may cause a general impairment in the identification of negative emotions.

A consistent finding across previous studies investigating emotion recognition in HD patients is that the recognition of positive emotions has been spared in the recognition of facial as well as vocal stimuli (e.g., Calder et al., 2010; Snowden et al., 2008, but see Sprengelmeyer et al., 1996). However, one limitation is that they have all included only a single positive emotion category (joy/happiness). Recent work, however, has shown that a range of positive states can be reliably communicated using non-verbal signals such as posture, voice, and touch (see Sauter, 2010). In particular, non-verbal vocalizations, such as laughs, cheers, and sighs, reliably signal joyful emotions including amusement, achievement, and pleasure (Sauter et al., 2010; Sauter and Scott, 2007), as well as negative emotions such as anger, disgust, fear and sadness. Non-verbal vocalizations thus provide a class of emotional signals in which the recognition of a range of both positive and negative emotions can be tested.

The aim of the current study was to examine emotion recognition in HD using several negative and positive emotions. In order to test the specificity of the impairment, we employed an emotion identification task using previously validated non-verbal affective vocalization stimuli of positive and negative emotions (Sauter et al., 2010; Sauter and Scott, 2007). This allowed us to address two hypotheses. One possibility is that the recognition of positive emotions is in fact spared in HD, as previous authors have found. Alternatively, previous studies may have failed to detect the impairment in the recognition of positive emotional stimuli because of the use of only a single class of positive emotional stimuli. This study examined the recognition of more than one type of positive emotional signal in HD.

\section{Methods}

\subsection{Participants}

The participants consisted of 14 genetically confirmed patients with HD [ 6 females, mean age $=51.29( \pm 7.69)$ years; mean years of formal education $=12.29( \pm 3.36)]$, and 15 control participants [ 7 female, mean age $=46.80( \pm 11.18)$ years; mean years of formal education $=12.53( \pm 2.64)$ ]. The patients and controls were matched for age $\left(t_{27}=-1.25, p>.2\right)$ and years of education $\left(t_{27}=.22, p>.8\right)$. Participants were native French speakers recruited through the biomarker program approved by the ethical committee of Henri Mondor Hospital, Créteil and gave informed consent to taking part in the study. The HD patients had no previous neurological or psychiatric history other than HD. None of the participants reported hearing problems in everyday life and their basic auditory perception was assessed using a computer beep; all participants comfortably perceived the test sound at a volume of 3.5 of 10 .

\subsection{Background neurological and neuropsychological assessment of HD patients}

The patients were at early stages of the disease, according to the Total Functional Capacity Scale (Shoulson, 1981), presenting with apparent but mild movement and cognitive impairments. None of the patients showed signs of depression. Data from the neurological and neuropsychological assessments of the HD patients are summarized in Table 1.

\subsection{Emotional vocalization stimuli}

The emotional vocalization stimuli were obtained from a previously validated set of affective non-verbal vocalizations, such as laughs, sighs, and screams, lasting approximately $1 \mathrm{sec}$ per stimulus (Sauter et al., 2010; Sauter and Scott, 2007). The categories included in the current study were from three positive emotions (achievement, amusement, and pleasure), and four negative emotions (anger, disgust, fear, and sadness). Ten tokens were used per emotion category, resulting in a set of 70 stimuli, with equal numbers of male and female vocalizations.

Table 1 - Background neuropsychological data for the HD patients, with published normal ranges when available.

\begin{tabular}{llcc} 
Test & \multicolumn{1}{c}{$\begin{array}{c}\text { Sub-scale/ } \\
\text { domain }\end{array}$} & $\begin{array}{c}\text { HD } \\
\text { patients }\end{array}$ & $\begin{array}{c}\text { Normal } \\
\text { cut-off }\end{array}$ \\
\hline \multirow{2}{*}{ UHDRS } & TFC (max 13) & $11.4(1.2)$ & - \\
& Motor (max 124) & $26.3(10.9)$ & - \\
& Behavioral (max 16) & $1.9(2.8)$ & - \\
MADRS & Depression (max 60) & $11.0(6.7)$ & $<15$ \\
FHVLT & Delayed recognition & $8.1(3.8)$ & $>9.7^{\mathrm{a}}$ \\
& (max 12) & & \\
MDRS & Total (max 144) & $128.9(11.7)$ & $>136$ \\
& Attention & $35.6(1.5)$ & $>31$ \\
& Initiation & $29.6(7.4)$ & $>28$ \\
\multirow{2}{*}{ TMT B } & Executive function & $161.9(58.7)$ & $>135$ \\
\hline
\end{tabular}

UHDRS = Unified Huntington's Disease Rating Scale (UHDRS; Huntington Study Group, 1996); Motor and Behavioral sub-scales and TFC (Total Functional Capacity) Scale (Shoulson, 1981), MADRS $=$ Montgomery and Asberg Depression Rating Scale (Montgomery and Asberg, 1979); FHVLT = French Hopkins Verbal Learning Test (Rieu, et al., 2006); MDRS = Mattis Dementia Rating Scale (Mattis et al., 1976; normal range norms from Guelfi, 1997); TMT B = Trail Making Test part B (Tombaugh, 2004).

a Cut-off was calculated as mean minus two standard deviations from the published control data $(11.3 \pm .8)$. 


\section{Procedure}

Participants were played the stimuli in a random order via headphones from a computer running a purpose-written Eprime script. After each stimulus, a list of the response alternatives was displayed on the screen [1: "accomplissement" (achievement), 2: "amusement" (amusement), 3: "colère" (anger), 4: "dégoût" (disgust), 5: "peur" (fear), 6: "plaisir" (pleasure), and 7: "tristesse" (sadness)], and participants were asked to judge the emotion expressed in the vocalization they heard. Participants responded by typing in a number on the keyboard, and no time limit was enforced.

\section{Statistical analysis}

In forced-choice tasks, performance for a particular category can be artificially inflated by the disproportionate use of that response. 'Hu' scores are unbiased hit rates that take into account each participant's use of the different response alternatives (Wagner, 1993). A score of zero denotes chance performance and a score of one perfect performance. Hu scores were calculated for patients' and controls' recognition rates for each emotion category, and tested against (Hu-score calculated-) chance level. Due to group sizes and heterogeneous population variances in the data, non-parametric analyses were employed for all analyses. Like Snowden et al. (2008) and several previous authors, we employed Mann-Whitney U tests for pairwise-comparisons, and Friedman tests for within-group comparisons. Further, like Snowden et al. (2008), correction procedures were not employed in order to avoid loss of power in these small samples.

\section{Results}

Both the HD patients and the controls were able to identify the emotions from the non-verbal vocalizations: they performed better than chance for the task overall (controls: $z=-3.41$, $p<.001$; HD patients: $z=-3.30, p<.001)$. However, the controls performed better than the HD patients [average performance for controls: $.52( \pm .12)$, and for patients: .28 ( \pm .16$), U=21, z=-3.67, p<.001]$.

Performance varied across the emotion types in the controls, $\left(\chi_{r}^{2}=29.5, p<.001\right)$, and there was a trend for variability across emotion categories in the patient group $\left(\chi_{r}^{2}=11.6, p=.07\right)$. Considering each emotion separately, both controls and HD patients performed above chance for all emotions [controls: $z=-3.35$ for achievement, $z=-3.41$ for amusement, $z=-3.41$ for pleasure, $z=-3.41$ for anger, $z=-3.42$ for disgust, $z=-3.41$ for fear, and $z=-3.42$ for sadness, all $p<.001$ ) and HD: $(z=-2.67$ for achievement; $z=-3.30$ for amusement; $z=-3.23$ for pleasure; $z=-3.23$ for anger; $z=-3.11$ for disgust; $z=-3.23$ for fear; and $z=-3.23$ for sadness; all $p<.001$, for achievement: $p=.004$ ]. Performance per emotion and group is shown in Fig. 1.

Recognition performance in HD patients was lower than in controls for achievement $(U=56.0, z=-2.1, p=.032)$, anger $(U=34.0, \quad z=-3.1, \quad p=.002), \quad$ disgust $(U=48.5, \quad z=-2.5$, $p=.012)$, fear $(U=14.5, \quad z=-4.0, \quad p<.001)$, and pleasure $(U=43.5, z=-2.7, p=.006)$, with a trend also for sadness $(U=60.5, z=-2.0, p=.051)$, and amusement vocalizations $(U=66.5, z=-1.7, p=.093)$. Importantly, recognition performance in HD patients was lower for the average of positive $(U=45.5, z=-2.6, p=.009)$ as well as the average of negative emotions $(U=21.5, z=-3.6, p<.001)$.

Confusion matrices are shown in Table 2. Errors were largely consistent across patients and controls, and included some cross-valence confusions. Common errors included sad sounds mistaken for pleasure, anger sounds classified as disgust, and achievement sounds perceived as signaling amusement.

\section{Discussion}

The results of this study show that patients with HD have reduced sensitivity in the identification of emotional signals overall, for positive as well as negative emotions. Importantly however, HD patients (as well as controls) were able to perform

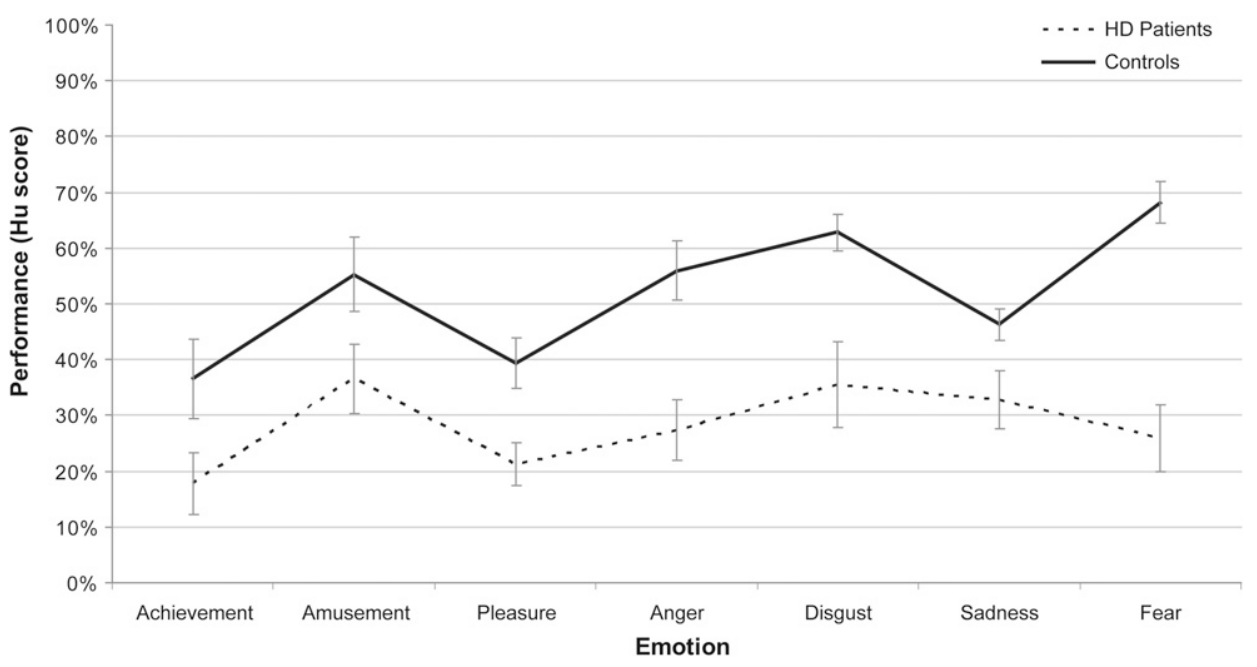

Fig. 1 - Performance (Hu scores) in the recognition of non-verbal vocalizations of emotions by Huntington's patients (dotted line) and controls (black line) in a 7-way forced-choice task. Standard error bars are shown for each category of vocalization for each group. 
Table 2 - Confusion matrices for controls and

Huntington's patients in percentages.

Stimulus Response

type

Achieve Amuse Plea Anger Disgust Fear Sadness

Controls $(n=15)$

$\begin{array}{lrrrrrrr}\text { Achieve } & 52.7 & 32.7 & 12.0 & .7 & .7 & 1.3 & .0 \\ \text { Amuse } & .7 & 85.3 & 13.3 & .0 & .0 & .0 & .7 \\ \text { Plea } & 13.3 & 2.0 & 69.3 & .7 & 6.0 & 1.3 & 7.3 \\ \text { Anger } & 12.0 & 6.0 & 1.3 & 58.7 & 14.7 & 6.7 & .7 \\ \text { Disgust } & 5.3 & 2.7 & 4.0 & 2.7 & 84.0 & 1.3 & .0 \\ \text { Fear } & 4.7 & 4.0 & 2.7 & 4.0 & 2.0 & 80.0 & 2.7 \\ \text { Sadness } & 6.7 & 2.0 & 19.3 & .7 & 8.7 & 2.7 & 60.0\end{array}$

HD patients $(n=14)$

$\begin{array}{lrrrrrrr}\text { Achieve } & 36.4 & 27.9 & 18.6 & 5.0 & 2.9 & 5.7 & 3.6 \\ \text { Amuse } & 10.0 & 67.9 & 15.0 & .7 & 1.4 & .7 & 4.3 \\ \text { Plea } & 17.1 & 7.1 & 48.6 & 3.6 & 12.1 & 6.4 & 5.0 \\ \text { Anger } & 12.1 & 7.9 & 2.9 & 38.6 & 17.1 & 19.3 & 2.1 \\ \text { Disgust } & 7.9 & 7.9 & 5.7 & 6.4 & 55.7 & 7.1 & 9.3 \\ \text { Fear } & 8.6 & 18.6 & 9.3 & 7.1 & 10.7 & 42.9 & 2.9 \\ \text { Sadness } & 10.7 & 6.4 & 20.7 & 4.3 & 7.9 & 5.0 & 45.0\end{array}$

Achieve $=$ Achievement, $\quad$ Amuse $=$ Amusement, Plea $=$ Pleasure Stimuli categories in rows and response categories in columns. All rows add to 100 . Correct responses are marked in bold.

the task at better-than-chance levels, overall and for each category of non-verbal emotional vocalizations. Additionally, the relative difficulty and confusion errors made by the patients largely mirrored those of the controls (see Table 2). This pattern suggests that the patients' impairment was not due to a failure to understand or perform the task, but rather a consequence of a reduced sensitivity to emotional cues in both positive and negative affective vocal signals. Although the current study did not include a control task testing higher order auditory processing for non-emotional stimuli, the general ability of the patients to perform the task and the similarity of their performance to that of the control participants, suggests that the emotion recognition impairment in HD was not due to primary auditory deficits. A previous study has also found intact performance in HD patients on auditory tasks including phoneme processing (Teichmann et al., 2009).

The results from the current study are consistent with previous work that has found impaired recognition of several negative emotions in HD (De Gelder et al., 2008; Johnson et al., 2007; Milders et al., 2003; Snowden et al., 2008), but our findings go beyond earlier research in demonstrating an impairment that extends to positive affective signals, thus highlighting the importance of examining the identification of positive emotion in more depth.

While further work will be needed to confirm whether HD patients are also impaired in the recognition of positive emotional signals in other modalities, our results suggest that the emotion recognition deficit in HD is not specific to a single emotion or to negative emotions. This finding lends some support to the hypothesis that emotion processing may be impaired at a general level in HD. Given that HD patients are impaired in the recognition of emotional cues from facial, bodily, and vocal signals (De Gelder et al., 2008; Snowden et al., 2008), this impairment likely occurs at post-perceptual processing levels.
Although little is known about HD patients' understanding of their own emotions, recent work has suggested that patients' general semantic representations are not impaired (Hayes et al., 2007; Snowden et al., 2008), highlighting the need to examine other mechanisms that may play a causal role in the emotion recognition impairment in HD. It is worth noting that, although our results suggest an overall impairment in the recognition of both positive and negative emotions, the impairment for certain emotions of both valence types appear to be particularly severe. The recognition of anger and fear was especially impaired in the HD patients compared to control participants in our study, like in Calder et al. (2010), who attributed this pattern to insensitivity to social disapproval in HD. However, insensitivity to social disapproval is unlikely to explain the strong impairment for pleasure signals also found in the current study.

By contrast, given the motor symptomatology in HD, one candidate mechanism may be affective motor representations, which have been suggested to underlie emotion recognition across modalities (Niedenthal, 2007).

Another important factor in understanding the underlying mechanisms in causing this impairment is the neuropathology associated with HD. Comparisons of the emotion recognition problems in HD patients with those of other patient groups with brain pathology affecting the striatum, such as Parkinson's Disease (PD), could inform our understanding of the role of striatal regions in emotion processing. Studies on PD patients' emotion recognition in the visual modality have yielded inconclusive results, with some finding an impairment (e.g., Kan et al., 2002), and others not (e.g., Adolphs et al., 1998).For auditory stimuli impaired recognition has been observed for a number of negative emotions (e.g., Lloyd, 1999; Pell, 1996), as well as for positive prosody (Pell and Leonard, 2003). A recent meta-analysis of emotion recognition in PD concluded that the identification of negative emotions is disproportionately impaired in this group of patients (Gray and Tickle-Degnen, 2010). However, no study to date has used more than one category of positive emotion with PD patients. Future comparisons of emotion recognition of a wide range of emotions in $\mathrm{HD}$ and $\mathrm{PD}$ patients may elucidate the contribution of the basal ganglia to emotion processing.

The results from the current study are the first to show an impairment of recognition of positive emotional signals from non-verbal vocal signals in HD. The results are consistent with recent findings that have failed to find a specific impairment in the recognition of disgust in $\mathrm{HD}$, and instead point to a global impairment of emotion recognition in HD, affecting both positive and negative emotional signals.

\section{Acknowledgments}

This work was supported by the GIS (Groupement d'Intérêt Scientifique) Institut Maladies Rares (A04159JS), the Dorothy M. and Maurice C. Shapiro Traveling Fellowship, and a Swiss National Funds post-doc grant to Iris Trinkler. The authors would like to thank Maryline Couette and Marie-Françoise Boissé for the general neuropsychological assessment, Katia Youssov for neurological assessment of the HD patients, and Severine Devignevielle for assistance with participant recruitment. 


\section{R E F E R E N C E S}

Adolphs R, Schul R, and Tranel D. Intact recognition of facial emotion in Parkinson's disease. Neuropsychology, 12(2): 253-258, 1998.

Calder AJ, Keane J, Young AW, Lawrence AD, Mason S, and Barker RA. The relation between anger and different forms of disgust: Implications for emotion recognition impairments in Huntington's disease. Neuropsychologia, 48(9): 2719-2729, 2010.

De Gelder B, Van den Stock J, De Diego Balaguer R, and BachoudLevi A- C. Huntington's disease impairs recognition of angry and instrumental body language. Neuropsychologia, 46(1): 369-373, 2008.

Gray HM and Tickle-Degnen L. A meta-analysis of performance on emotion recognition tasks in Parkinson's Disease. Neuropsychology, 24(2): 176-191, 2010.

Gray JM, Young AW, Barker WA, Curtis A, and Gibson D. Impaired recognition of disgust in Huntington's disease gene carriers. Brain, 120(11): 2029-2038, 1997.

Guelfi JD. L'évaluation clinique standardisée en psychiatrie. Boulogne: Editions Médicales Pierre Fabre, 1997.

Hayes CJ, Stevenson RJ, and Coltheart M. Disgust and Huntington's disease. Neuropsychologia, 45(6): 1135-1151, 2007.

Huntington Study Group. The unified Huntington's disease rating scale: Reliability and consistency. Movement Disorders, 11(2): 136-142, 1996.

Johnson SA, Stout JC, Solomon AC, Langbehn DR, Aylward EH, Cruce $C B$, et al. Predict-HD Investigators of the Huntington Study Group. Beyond disgust: Impaired recognition of negative emotions prior to diagnosis in Huntington's disease. Brain, 130(7): 1732-1744, 2007.

Kan Y, Kawamura M, Hasegawa Y, Mochizuki S, and Nakamura K. Recognition of emotion from facial, prosodic and written verbal stimuli in Parkinson's disease. Cortex, 38(4): 623-630, 2002.

Lloyd AJ. Comprehension of prosody in Parkinson's disease. Cortex, 35(3): 389-402, 1999.

Mattis S, Bellak L, and Karasu TB. Mental status examination for organic mental syndrome in elderly patients. In: Geriatric Psychiatry. New York: Grune \& Straton, 1976: 77-121.

Milders M, Crawford J, Lamb A, and Simpson S. Differential deficits in expression recognition in gene-carriers and patients with Huntington's disease. Neuropsychologia, 41(11): 1484-1492, 2003.

Montgomery SA and Asberg M. A new depression scale designed to be sensitive to change. The British Journal of Psychiatry, 134: 382-389, 1979.

Niedenthal PM. Embodying emotion. Science, 316(5827): 1002-1005, 2007.

Pell MD. On the receptive prosodic loss in Parkinson's disease. Cortex, 32(4): 693-704, 1996.

Pell MD and Leonard CL. Processing emotional tone from speech in Parkinson's disease: A role for the basal ganglia. Cognitive, Affective \& Behavioral Neuroscience, 3(4): 275-288, 2003.

Rieu D, Bachoud-Levi A-C, Laurent A, Jurion E, and Dalla Barba G. [French adaptation of the Hopkins Verbal Learning Test]. Revue Neurologique, 162(6-7): 721-728, 2006.

Sauter DA. More than happy: The need for disentangling positive emotions. Current Directions in Psychological Science, 19(1): 36-40, 2010.

Sauter DA, Calder AJ, Eisner F, and Scott SK. Perceptual cues in non-verbal vocal expressions of emotion. Quarterly Journal of Experimental Psychology, 28: 1-22, 2010.

Sauter DA and Scott SK. More than one kind of happiness: Can we recognize vocal expressions of different positive states? Motivation and Emotion, 31(3): 192-199, 2007.

Shoulson I. Huntington disease: Functional capacities in patients treated with neuroleptic and antidepressant drugs. Neurology, 31(10): 1333-1335, 1981.

Snowden J, Austin N, Sembi S, Thompson J, Craufurd D, and Neary D. Emotion recognition in Huntington's disease and frontotemporal dementia. Neuropsychologia, 46(11): 2638-2649, 2008.

Sprengelmeyer R, Young AW, Calder AJ, Karnat A, Lange H, Hömberg V, et al. Loss of disgust: Perception of faces and emotions in Huntington's disease. Brain, 119(5): 1647-1665, 1996.

Teichmann M, Darcy I, Bachoud-Lévi AC, and Dupoux E. The role of the striatum in phonological processing: Evidence from early stages of Huntington's disease. Cortex, 45: 839-849, 2009.

Tombaugh TN. Trail Making Test A and B: Normative data stratified by age and education. Archives of Clinical Neuropsychology, 19(2): 203-214, 2004.

Wagner H. On measuring performance in category judgment studies of nonverbal behavior. Journal of Nonverbal Behavior, 17(1): 3-28, 1993. 